\title{
The effect of ferulic acid on experimental traumatic brain damage in rats
}

\author{
Erbil $\mathrm{G}^{1}$, Sacik $\mathrm{U}^{1}$, Yilmaz $\mathrm{F}^{1}$, Kisaoglu $\mathrm{H}^{1}$, Erbayraktar $\mathrm{Z}^{2}$, Pekcetin $\mathrm{C}^{1}$, Ozogul $\mathrm{C}^{3}$ \\ Dokuz Eylul University, Department of Histology and Embryology, Faculty of Medicine, Izmir, Turkey. \\ drfilizyilmaz@gmail.com
}

\begin{abstract}
AIM: Traumatic brain injury is an important social health problem due to the fact that young adults are more likely to be affected, and advanced functional limitations are observed in survivors. In this study, we aimed to investigate the protective effect of ferulic acid in an experimental trauma model.

MATERIAL AND METHODS: This study was performed in March 2016 at Dokuz Eylül University Experimental Animal Laboratory. Subjects were randomly divided into 4 groups Control, Ethyl Alcohol, Trauma, Trauma/Ferulic Acid groups. For histological findings, Cresyl violet; for immunohistochemical analysis, TUNEL and Active Caspase-3 staining were used. For biochemical analysis, Superoxide dismutase, Malondialdehyde, and Glutathione values were examined.

RESULTS: The application of ferulic acid has been shown to primarily reduce neuronal apoptosis, the levels of free radicals, and to effect oxidant/antioxidant balance positively by increasing the levels of antioxidants, such as Superoxide dismutase and Glutathione that are developed due to brain damage. Our study group has shown that ferulic acid decreased nerve tissue pathologies after generated brain trauma compared to injury groups. CONCLUSION: Addition of ferulic acid to the traditional head trauma treatment has the strength, and ability to increase the rate, and percentage of healing (Tab. 2, Fig. 4, Ref. 28). Text in PDF www.elis.sk.

KEY WORDS: ferulic acid, head trauma, hippocampus, prefrontal cortex.
\end{abstract}

\section{Introduction}

Traumatic brain injury (TBI) is a brain injury that occurs when the head suddenly, and violently strikes an object or the object causes damage to the brain tissue by piercing the skull (1, 2). TBI is one of the leading causes of death, and disability, and often causes post-traumatic epilepsy (PTE) development (3). TBI can be classified as a closed head injury, open head injury, and penetrating head injury.

Closed and open head injury terms define collisions between the head, and a blunt object. Closed head trauma is a diffuse axonal injury characterized by intact dura, and is due to the mechanism of acceleration-deceleration $(2,4)$. Diffuse axonal injury constitutes $40-50 \%$ of TBI causes and is the most important cause of loss of consciousness after trauma. Open head trauma defines injuries in which the dura is breached. Penetrating head trauma is an injury caused by firearm in which a foreign body penetrates the dura, and enters the brain or by knife injuries. The prognosis is highly vari-

${ }^{1}$ Dokuz Eylul University Faculty of Medicine, Department of Histology and Embryology, Izmir, Turkey, ${ }^{2}$ Dokuz Eylul University Faculty of Medicine, Department of Biochemistry, Izmir, Turkey, and ${ }^{3}$ University of Kyrenia Faculty of Medicine, Department of Histology and Embryology, Kyrenia, Northern Cyprus

Address for correspondence: F. Yilmaz, Dokuz Eylul University, Department of Histology and Embryology, Faculty of Medicine, 35340 Izmir, Turkey.

Phone: +90.232 .4124595 , Fax: +90.232 .4129798$ able depending on the shape and severity of the injuries (4). The terms mild, moderate, and severe TBI reveal TBI severity $(5,6)$. The mechanisms leading to TBI can be classified as primary and secondary injuries. Primary mechanisms occur at the time of the injury whereas secondary injury is the response of the organism to the primary mechanism, and both may be focal or diffuse. Focal injuries occur with direct impacts, and diffuse injuries occur with acceleration-deceleration, and rotational forces (5).

Primary Injury: Primary injuries occur as a result of cerebral contusion, intracranial hematoma, (epidural, subdural, subarachnoid), diffuse axonal injury, and acceleration-deceleration. Cortical contusions arise in specific regions of the base of the head depending on the characteristics of the bone structure.

Contusions are associated with subarachnoid haemorrhage and following epileptic attacks $(2,5)$. Axonal stretch injuries are seen in midline tissues, cerebral cortex parasagittal white matter, corpus callosum, pontine-mesencephalic junction near the superior cerebral peduncle $(7,8)$.

Diffuse axonal injuries can only be identified microscopically. At the same time; diffuse axonal injury can also be observed in high-speed collisions. Though the traumatic diffuse axonal injury is being associated with cerebral atrophy, the relationship of volume of white matter, and afferent, and efferent axonal pathways with traumatic axonal injury is unknown (8).

Secondary Injury: Secondary damage formation is a complex chain of intertwining events. After TBI, the first stages of cerebral injury are characterized by a disturbance of cerebral blood flow 
$(\mathrm{CBF})$, and metabolism regulation with direct cell damage and thereafter by cell death $(9,10,11)$.

Events such as hypotension, increased intracranial pressure, cerebral perfusion pressure reduction, neural cell membrane damage are seen. Hypotension or arterial oxygen pressure of $<60 \mathrm{mmHg}$ immediately after trauma increases morbidity and mortality (2). Secretion of glutamate in cellular level with response to injury in TBI increases neuronal migration of calcium and sodium by activating AMPA and NMDA receptors.

Neuronal energy stores are consumed to retain sodium/potassium ratio, free radicals, and oxidants are released with neuronal cell membrane damage, and these cause an increase in glutamate, a continuation of the loop. At the same time, enzymes, such as caspase (proteolytic enzyme) lead to neuronal apoptosis by increasing intracellular calcium (2).

Ferulic acid found in seeds, and leaves of plants is a phenolic acid. It is found quite a lot especially in plants such as rice, wheat, oat, orange, pineapple, artichokes. In addition, the chemical structure is similar to the structure of strong curcumin, and is a member of the hydroxycinnamic acid family (12). In studies, it was shown that ferulic acid is an antioxidant neutralizing free radicals such as DNA-damaging superoxide, nitric oxide, and hydroxyl radicals $(13,14)$. Ferulic acid absorption takes place throughout the entire stomach, and bowel (15). While metabolism takes place in the liver, excretion is renal (16). While more than $1929 \mathrm{mg} / \mathrm{kg}$ creates toxic effect for rats, the amount that should be taken daily is $150-250 \mathrm{mg} / \mathrm{kg}(16-24 \mu \mathrm{mol} / \mathrm{kg}$ body weight) $(15,16)$. In earlier studies it was shown that ferulic acid is present in tomato $(6 \mathrm{mg} / 100 \mathrm{~g})$, pasta $(12 \mathrm{mg} / 100 \mathrm{~g})$, white wheat bread $(8.2 \mathrm{mg} / 100 \mathrm{~g})$, broccoli $(4.1 \mathrm{mg} / 100 \mathrm{~g})$, grapefruit $(11 \mathrm{mg} / 100$ g), banana (5.4 mg/100 g) (16).

In studies, it was shown that ferulic acid is an antioxidant neutralizing free radicals such as DNA-damaging superoxide, nitric oxide, and hydroxyl radicals $(13,14)$. Our aim in this study is to investigate the histochemical, immunohistochemical, and biochemical effects of powerful antioxidant ferulic acid on TBI that was created in the rats depending on the trauma model.

\section{Material and methods}

\section{Animals}

All experimental procedures were approved by the Dokuz Eylul University Local Ethical Committee of Animal Experiments (Protocol No. 01/2015). This study was done in March 2016 at Dokuz Eylül University Experimental Animal Laboratory.

In this study, a total of twenty-eight Wistar strain male rats were used that are obtained from the Research Unit of Dokuz Eylül University Faculty of Medicine. Wistar strain were chosen due to its presence in Dokuz Eylül University Faculty of Medicine Experimental Animal Laboratory, and is compatible with the literature. All subjects were housed in the standard animal room, and cages at $20-22{ }^{\circ} \mathrm{C}$ room temperature with $50-60 \%$ relative
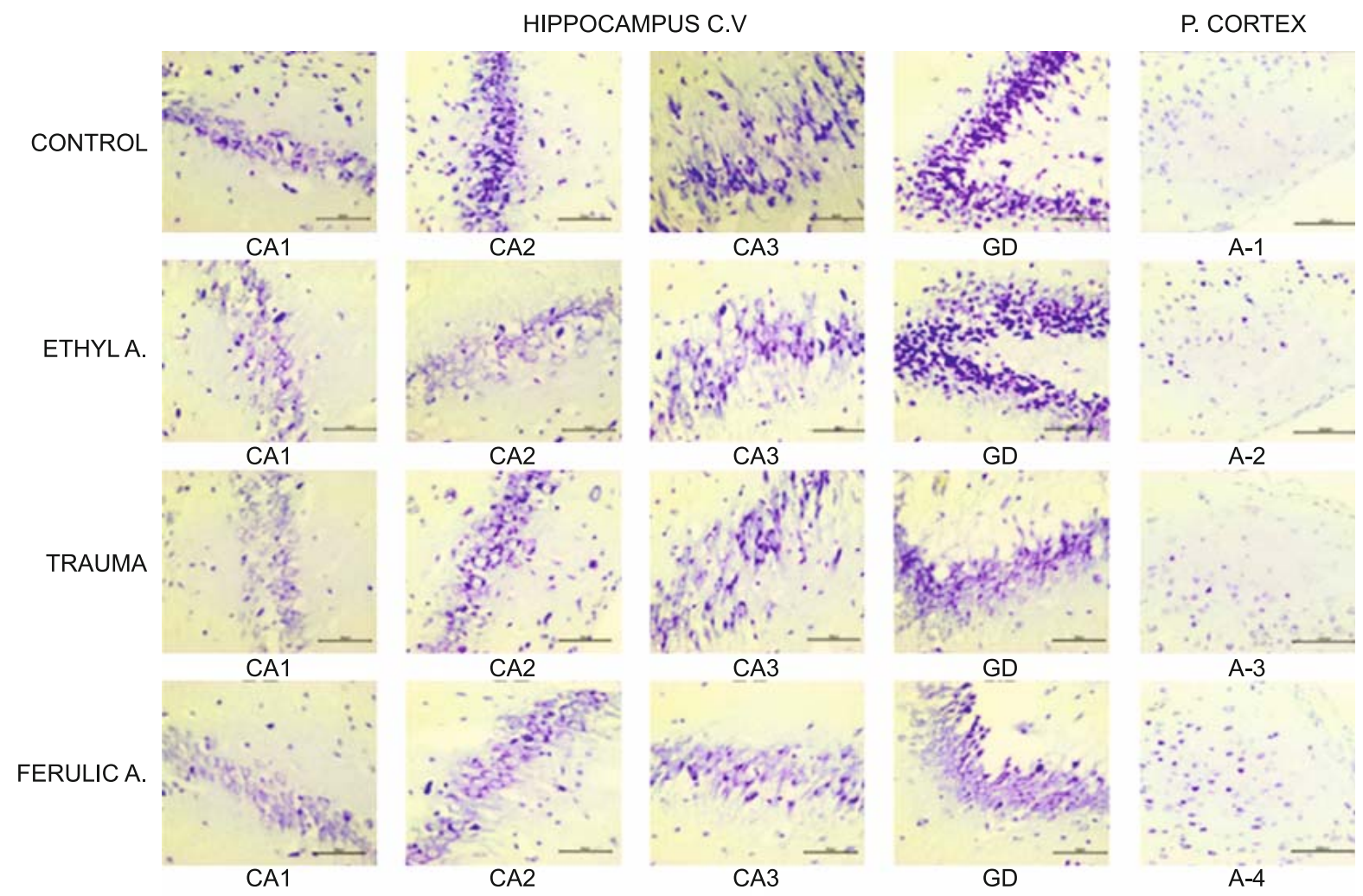

Fig. 1. The image of the hippocampus, and prefrontal cortex of brain tissue stained with C.V. Hippocampus [CA1, CA2, CA3, GD] (x40), prefrontal cortex [A 1-4] (x20). 
humidity in 12/12 hours of dark/bright periods and fed with rested tap water, and standard pellet feed in Experimental Animal Laboratories until the end of the experiment (ad libitum).

\section{Experimental design}

The study was a prospective experimental study. Twenty-eight rats were divided randomly into four groups. Control group $(\mathrm{C}$, $\mathrm{n}=7$ ), Ethyl Alcohol Group (E.A, $\mathrm{n}=7$ ), Trauma Group (, $\mathrm{n}=$ 7), Trauma/Ferulic acid group (T/F.A n =7). $50 \mathrm{mg} / \mathrm{kg}$ Ketamine $+10 \mathrm{mg} / \mathrm{kg}$ Xylazine was used intraperitoneally for anaesthesia of the subjects. After anaesthesia, the trauma model with weight drop was applied. After shaving the skull skin under anaesthesia, a metal disc was placed in the centre by pulling back the periosteum applying midline incision. $100 \mathrm{~cm}$ long contusion pipe created by piercing its surface with $10 \mathrm{~cm}$ intervals has been kept perpendicular on the round metal disk pressed against the parietal bone. To create brain injury the $300 \mathrm{~g}$ weight was dropped from $100 \mathrm{~cm}$ height with stereotaxic coordinates of $3 \mathrm{~mm}$ front $\mathrm{x} 2 \mathrm{~mm}$ side onto the skull (1). 48 hours after finishing the application of head trauma lives of all subjects in the groups were ended with cervical dislocation under ketamine-xylazine anaesthesia. The extracted brain tissue of sacrificed subjects' left hemispheres were used for biochemical analysis, and right hemispheres were used for the histological analysis.

Immediately after trauma application (Ferulic acid, $100 \mathrm{mg} / \mathrm{kg}$ (sc-204753, Santa Cruz, USA) dissolved in $400 \mu$ l ethyl alcohol to be $100 \mathrm{mg} / \mathrm{kg}$ was administered intraperitoneally (17).

\section{Histological methods}

Tissue samples were fixed in $10 \%$ formaldehyde for 48-hours. Then by applying routine tissue processing procedure, the tissues were embedded in paraffin blocks. $5 \mu \mathrm{m}$ coronal sections were obtained from the blocks according to the rat brain atlas (Rat Atlas of Paxinos and Watson) from 9, 11 th planes for prefrontal cortex, from 21, 23, 25th planes for the hippocampus. Some of the sections were stained with Cresyl violet $(\mathrm{CV})$ in order to examine tissue histology, and some with immunohistochemistry kits (Tunel, Active Caspase-3) for determination of apoptotic cells.

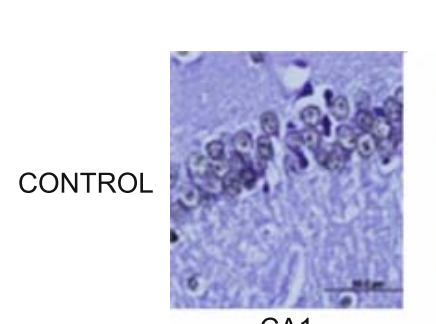

CA1

ETHYLA

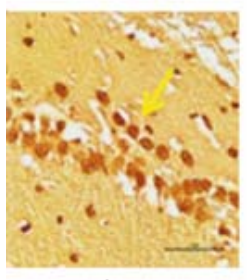

CA1

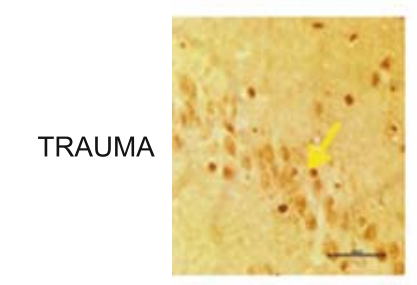

CA1

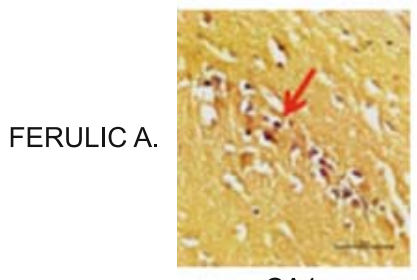

CA1

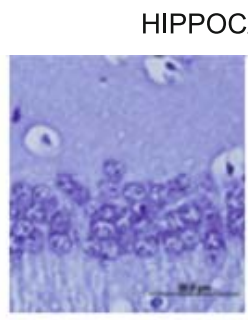

CA2

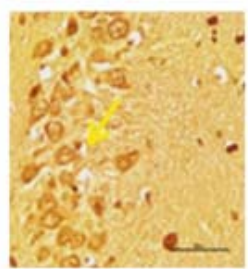

CA2

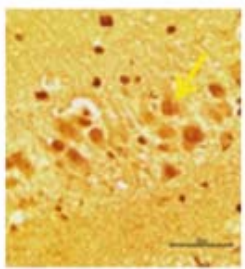

CA2

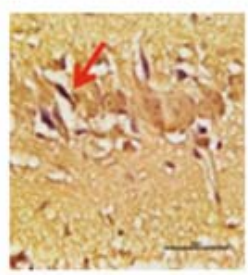

CA2

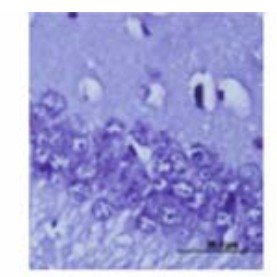

CA3

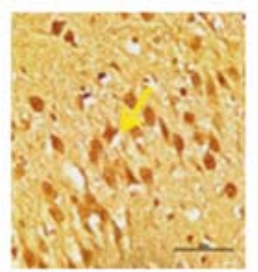

CA3

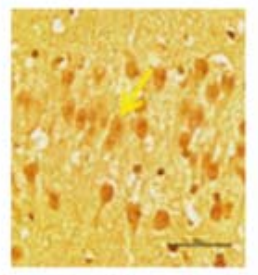

CA3

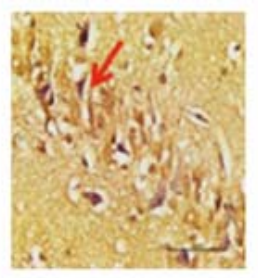

CA3

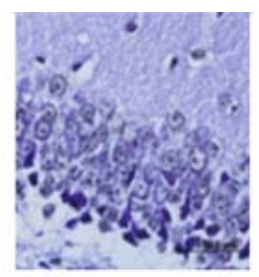

GD

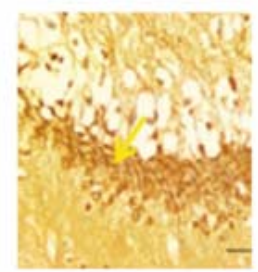

GD

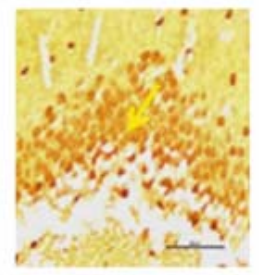

GD

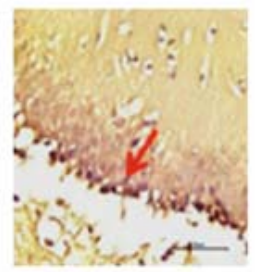

GD

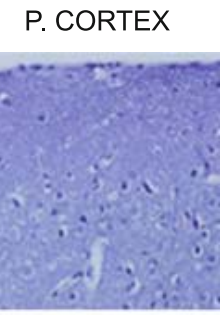

A-1

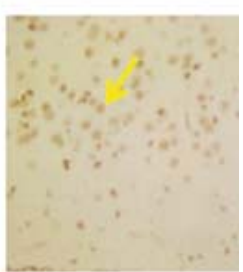

A-2

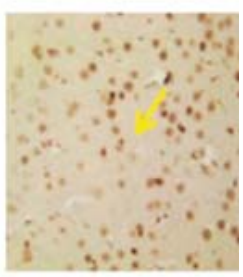

A-3

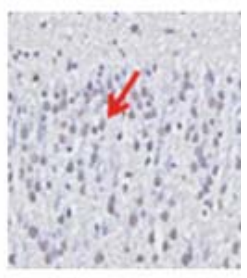

A-4

Fig. 2. The image of the hippocampus, and prefrontal cortex of brain tissue stained with Tunel. Hippocampus [CA1, CA2, CA3, GD] (x40), prefrontal cortex [A 1-4] (x20). (Yellow arrows show Tunel positive cells of damage groups, red arrows show normal cells of the treatment group). 


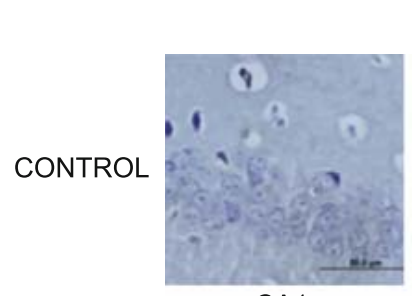

CA1

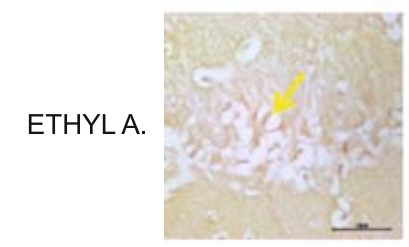

CA1

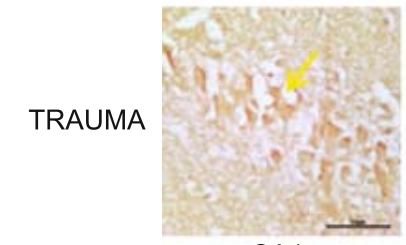

CA1

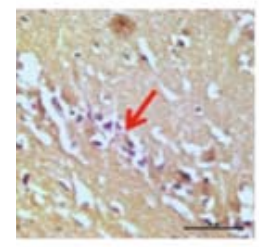

CA1

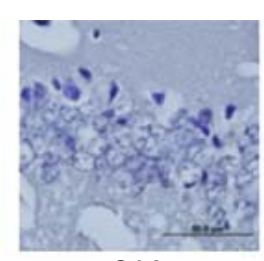

CA2

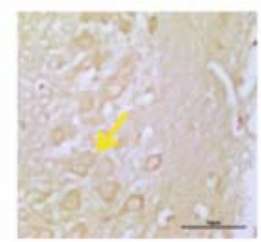

CA2

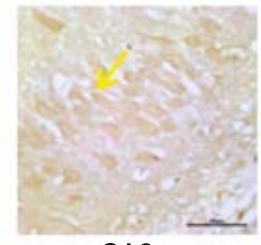

CA2

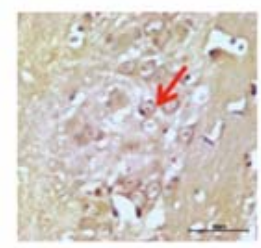

CA2

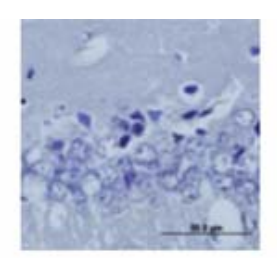

CA3

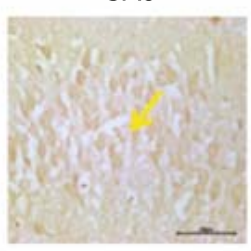

CA3

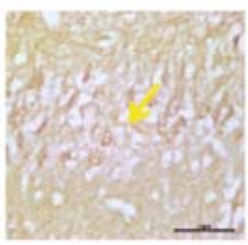

CA3

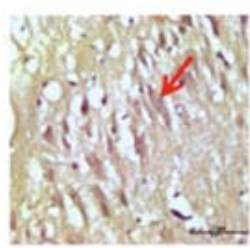

CA3
P. CORTEX

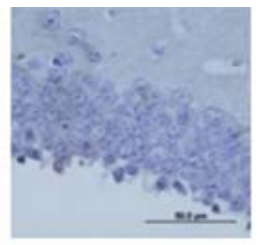

GD

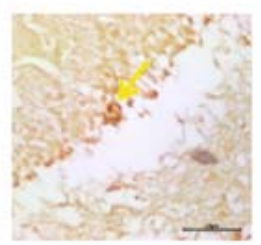

GD

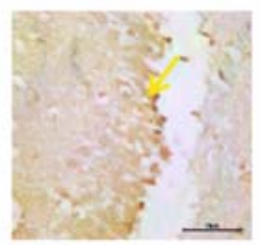

GD

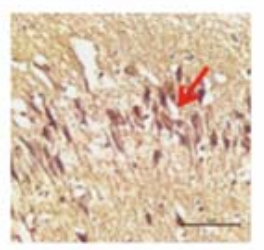

GD

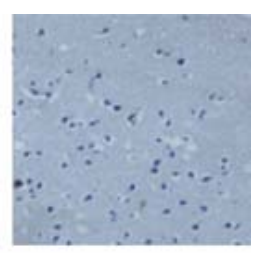

A-1

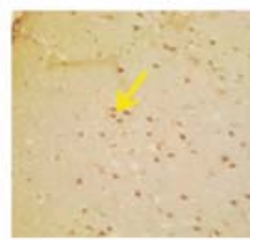

A-2

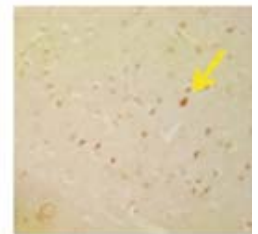

A-3

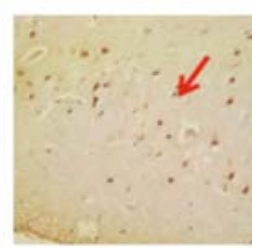

A-4

Fig. 3. The image of the hippocampus, and prefrontal cortex of brain tissue stained with Caspase. Hippocampus [CA1, CA2, CA3, GD] (x40), prefrontal cortex [A 1-4] (x20). (Yellow arrows show Tunel positive cells of damage groups, red arrows show normal cells of treatment group).

\section{Image analysis methods}

The images of cresyl-violet stained sections were obtained using computer-assisted image analysis system which includes light microscopy (Olympus BX-51 Tokyo, Japan), and high-resolution video camera (Olympus DP-71, Japan). Counting of the prefrontal cortex, parietal cortex, and hippocampal neurons was made in the monitor displayed by 20x Olympus lenses aided by counting frame of $15800 \mu \mathrm{m}^{2}$ in the right hemisphere. The counting frame was placed 3 times randomly over the image analysis system monitor, and evaluated statistically by obtaining the averages of the result (11).

\section{Immunohistochemical methods}

To detect DNA fragmentation in cell nuclei, terminal deoxynucleotidyl transferase-mediated dUTP-end labelling (TUNEL; In Situ Cell Death Detection Kit, Roche, Manheim, Germany In) kit was applied to $5 \mu \mathrm{m}$ paraffin sections. $5 \mu \mathrm{m}$ sections were put to slides covered with poly-lysine. After deparaffinization of obtained sections, they were dehydrated in graded alcohol series. After dehydration, they were treated with Proteinase K for 15 minutes in $37{ }^{\circ} \mathrm{C}$ incubator. After this procedure, tissue sections were washed with PBS. Then they were incubated with fluorescent labelled deoxy-UTP, and TdT in $37^{\circ} \mathrm{C}$ incubator for $60 \mathrm{~min}$. Tissue sections by washing again with PBS were incubated with POD solution for $30 \mathrm{~min}$ in $37{ }^{\circ} \mathrm{C}$ incubator. After the sections were washed with PBS again, they were stained with DAB (Roche Diagnostics, Mannheim, Germany). After DAB staining counterstaining method was performed with Hematoxylin staining. To calculate the apoptotic index, cells indicating apoptotic morphology were calculated as percentage by counting cells in the prefrontal cortex, the hippocampus of the right hemisphere.

Tab. 1. \% values of average cell counts in C.V. and immunostained prefrontal cortex.

\begin{tabular}{lccc}
\hline & \multicolumn{3}{c}{ Prefrontal cortex } \\
\cline { 2 - 4 } & C.V & Tunel & Caspase 3 \\
\hline C $(n=7)$ & $5.42 \pm 0.14$ & $1.71 \pm 0.9$ & $1.95 \pm 0.1$ \\
E.A $(n=6)$ & $4.55 \pm 0.4^{*}$ & $3.33 \pm 0.07^{*}$ & $3.38 \pm 0.13^{*}$ \\
T $(n=7)$ & $3.52 \pm 0.99^{*}$ & $4.85 \pm 0.09^{*}$ & $3.34 \pm 0.01^{*}$ \\
T - F.A $(n=6)$ & $4.88 \pm 0.26^{\#}$ & $2.66 \pm 0.07^{\#}$ & $2.49 \pm 0.04^{\#}$ \\
\hline
\end{tabular}

$* \mathrm{p}<0.05$, When control, and damage groups are compared, ${ }^{*} \mathrm{p}<0.05$, When damage groups, and $\mathrm{T}-\mathrm{F}$.A group are compared 
$5 \mu \mathrm{m}$ sections were put to slides covered with poly-lysine for active caspase-3 (bs-0081R, BIOSS, polyclonal antibody, USA). After deparaffinization of obtained sections, they were dehydrated in graded alcohol series. After dehydration process, they were treated with Proteinase $\mathrm{K}$ for 15 minutes in $37^{\circ} \mathrm{C}$ incubator. After this procedure, tissue sections were washed with PBS. Then preparations were treated with $\mathrm{H}_{2} \mathrm{O}_{2}$ for 30 min. Sections were washed again with PBS, and incubated with active caspase- 3 that is a primary antibody with $1: 100$ ratio at $+4{ }^{\circ} \mathrm{C}$ for 18 hours. Then preparations were kept in incubator with biotinylated IgG (secondary antibody) in $37^{\circ} \mathrm{C}$ with three stages being $30 \mathrm{~min}$ each (Invitrogen-Plus a Broad Spectrum, 85-9043). Apoptotic cells in the right hemisphere were calculated as percentage with similar background staining.

\section{Biochemical analysis}

Superoxide dismutase (SOD), glutathione peroxidase (GSH-Px) enzyme activities were measured in the supernatant, malondialdehyde (MDA) level was measured in homogenate spectrophotometrically in brain tissues.

Tissues were homogenized with the homogenizer (U1tra Turrax Type, T25-B, IKA Labortechnic, Germany) in 0.15 $\mathrm{M} \mathrm{KCl}$ solution of at $16000 \mathrm{rpm}$ for 3 minutes. Homogenization was held in an ice container. The supernatant was obtained by centrifugation of the homogenate at $5000 \mathrm{~g}$ for 1 hour $(+4$ ${ }^{\circ} \mathrm{C}$ ), and was kept at $-40{ }^{\circ} \mathrm{C}$ until the time of analysis (1 week). SOD, GSH-Px enzyme activities were measured in the supernatant, MDA levels were measured spectrophotometrically in the homogenate.
SOD enzyme was determined with the method modified by Sun et al (17). The basic principle of this method depends on the reading of blue colored compound at $505 \mathrm{~nm}$ that is formed by reduction of nitroblue tetrazolium (NBT) by superoxide producer xanthine-xanthine oxidase system. SOD activity was expressed as unit/g $(\mathrm{U} / \mathrm{g})$ tissue protein.

GSH-Px activity was determined using the method of Paglia et al (18). GSH-Px catalysis the oxidation of reduced glutathione (GSH) to oxidized glutathione (GSSG) in the presence of hydrogen peroxide. GSSG formed by GSH-Px's in the environment with hydrogen peroxide is reduced to GSH with the help of glutathione reductase, and NADPH. GSH-Px activity was defined with reading of absorbance decrease during oxidation of NADPH to $\mathrm{NADP}^{+}$at $340 \mathrm{~nm}$. GSH-Px activity was expressed as unit/g (U/g) tissue protein.

It was carried out using the Esterbauer method, which is a lipid peroxidation measurement method (19). Malondialdehyde reacting with thiobarbituric acid at $90-95{ }^{\circ} \mathrm{C}$ creates a pink chromogen. Fifteen minutes later, the samples were cooled rapidly, and their absorbances were read spectrophotometrically at $532 \mathrm{~nm}$. The obtained values were expressed as $\mathrm{nmol} / \mathrm{g}$ protein.

Tissue protein level in the homogenates of tissue samples was measured with the method determined by Lowry et al (19).

\section{Statistical analysis}

Statistical analysis of the Data obtained in the process of the study was done with SPSS (Statistical Package for Social Sciences) 23.0 computer package program. Mean, and standard deviation were determined in the evaluation. The difference between the groups was analyzed with Kruskal-Wallis test, from which group the difference between the groups is originated was analyzed with Mann-Whitney U test (11).

\section{Results}

The analyses made in the study were divided into 3 parts as histochemical, immunohistochemical, and biochemical. Two of the subjects died in the study, one from the ethyl alcohol, and other from the treatment group. The findings were evaluated from 26 rats.

While tissues belonging to the control group were normal in structure, it was seen that there was a significant decrease in the number of cells in CA1, CA, CA3, and GD areas of hippocampus, and prefrontal cortex in the injury created groups (E.A, and T), on the contrary in the group treated with F.A these values were increased significantly compared to the other injury groups, and were close to the control group ( $p<0.05)$ (Fig. 1).

In this study, statistical analysis was done by examining the number of TUNEL positive cells in x20 magnification for the prefrontal cortex, and hippocampus (Tab. 2) $(\mathrm{p}<0.05)$. It was observed that the number of positive cells was significantly increased in damage groups compared to the control group, when damage groups were examined among themselves, no significant difference in the numbers of positive cells was seen. Significant reduction in the number of positive cells was seen in the group treated with ferulic acid (T-F.A) compared to the damage groups (Fig. 2). 
(A)

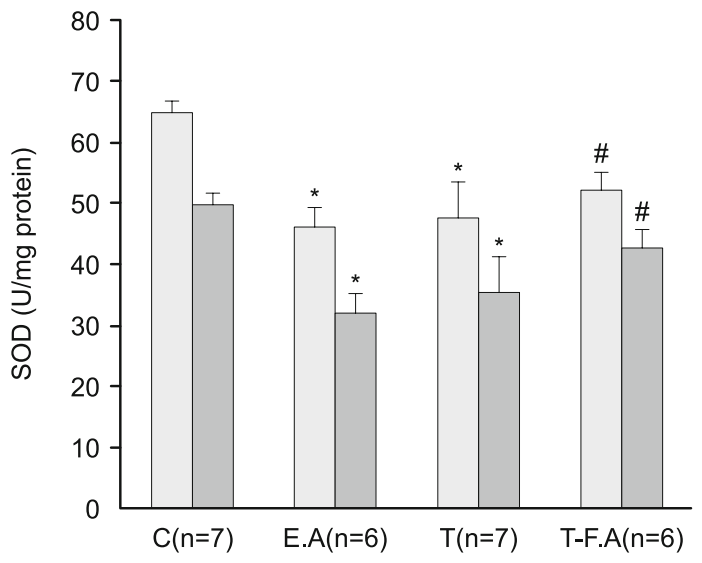

B

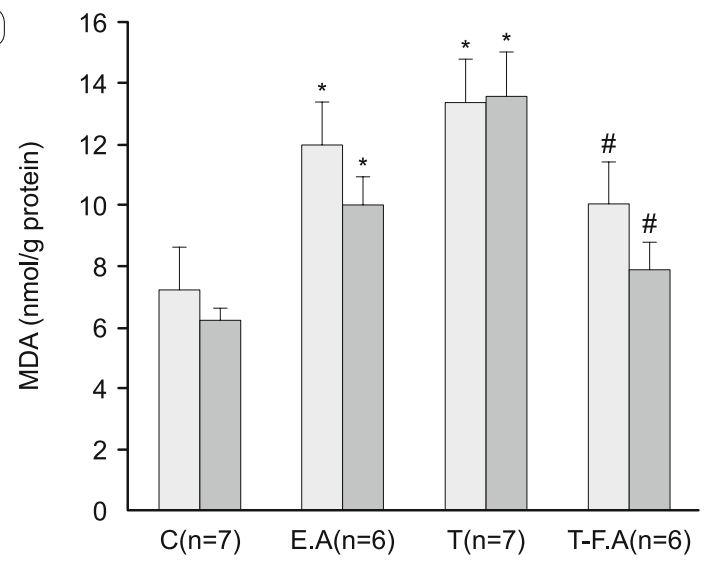

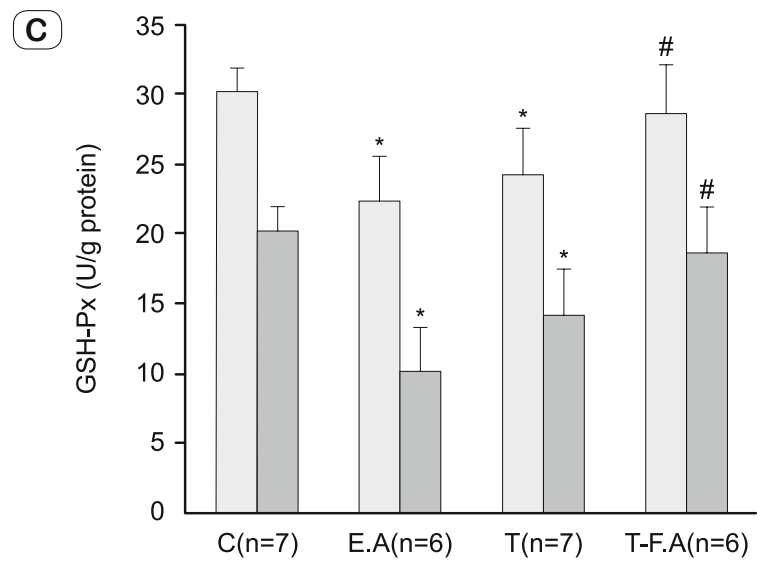

hypocampus

prefrontal c.

Fig. 4. Average (A) SOD, (B) MDA and (C) GSH values of the hippocampus and prefrontal cortex according to the groups (* $p<0.05$ control vs trauma, \# $p<0.05$ trauma vs trauma - ferulic acid).

In the study, statistical analysis was done by examining the numbers of positive cells at $\mathrm{x} 20$ magnification for prefrontal cortex (Tab. 1), and hippocampus (Tab. 2) $(\mathrm{p}<0.05)$. While significant increase in number of positive cells was observed in damage groups compared to control group, no significant difference was seen in terms of positive cells when damage groups were examined among themselves. Significant reduction in the number of positive cells was observed in the group treated with ferulic acid (T/F.A) compared to the damage groups (Fig. 3).

It was observed that SOD activity of the hippocampus and prefrontal cortex was reduced significantly in all other groups compared to the control group. While the decreases in damage groups were accepted as significant compared to the control group, the difference between the groups was not considered as significant when damage groups were examined among themselves. The increase in SOD activity was determined significant in treated T/F.A group compared to damage groups $(\mathrm{p}<0.05)$ (Fig. 4A).

It was observed that MDA activity of hippocampus, and prefrontal cortex was increased significantly in all other groups compared to the control group. While the increases in damage groups were accepted to be significant compared to the control group, the difference between the groups was not considered to be significant when damage groups were examined among themselves. The increase in MDA activity was determined to be significant in treated T/F.A group compared to damage groups $(\mathrm{p}<0.05)$ (Fig. 4B).

It was observed that SOD activity of the hippocampus, and prefrontal cortex was reduced significantly in all other groups compared to the control group. While the decreases in damage groups were accepted as significant compared to the control group, the difference between the groups was not considered as significant when damage groups were examined among themselves. The increase in GSH activity was determined to be significant in treated T/F.A group compared to damage groups $(\mathrm{p}<$ 0.05) (Fig. 4C).

\section{Discussion}

TBI is a pathological condition having debilitating, and possibly lethal properties as a result of primary, and secondary dam- 
age. Brain swelling, resulting in neuronal death as a result of increased intracranial pressure, and decreased cerebral perfusion, has an important role in secondary brain damage. TBI is not only a pathophysiologic event but is a complex disease process (1). It causes structural damage due to both primary, and secondary damage mechanisms, and functional deficiencies (20).

MDA is a substance passing through the cell membrane easily, and can accumulate in the cell (21). It can affect the passage of ions by causing polymerization of membrane components (22). Membrane permeability in cells can vary depending on lipid peroxidation Thus, a decrease in the enzymes found in the structure of the membrane occurs and the protein synthesis mechanism is inhibited (23). SOD is an enzyme that protects the cell from free oxygen radicals. SOD tries to eliminate the harmful effects by converting free oxygen radicals to hydrogen peroxide (24).

In the studies, histological images of C.V staining were shown, statistical analysis was made by neuron counting, and immunohistochemically, positive cell density was shown in the hippocampus (CA1, CA2, CA3, GD), and prefrontal cortex areas of the brain by Tunel, and caspase- 3 immunostaining (25). In the study that we have performed, parallel to these studies, similar results with literature were obtained for all three stainings that are C.V, Tunel, and caspase-3. In areas of hippocampus CA1, CA2, CA3, GD, and prefrontal cortex that we performed counting, a significant decrease in damage groups compared to control group according to C.V staining was seen, and as in the treatment group, significant increase were observed compared to damage groups. Similarly, Tunel, and caspase- 3 positive cells were quite increased in these areas of the hippocampus, and prefrontal cortex in damage groups compared to the treatment group, it showed a significant decrease.

In the studies SOD, MDA, and GSH values of the hippocampus (CA1, CA2, CA3, GD), and prefrontal cortex areas were shown by biochemical examination $(25,26)$. The results of our study group showed that similarly, SOD value in the hippocampus, and prefrontal cortex areas of the brain was reduced in damage group compared to control group whereas in treated group it was observed that this rate was increased significantly. Similarly, when GSH levels were examined, GSH values were observed to increase significantly in treated groups compared to damage groups. When values were examined in terms of MDA, is was seen that MDA levels increased in damage groups compared to the control group, and decreased in treated groups as to be close to the control.

As a result, like in previous studies, it was also shown with our study that the trauma model of the brain is a process that creates damage. Head trauma may result in significant symptoms and poor prognosis $(27,28)$. Given the damage was done in the brain by trauma, we showed that it causes reduction in numbers of neurons histologically, and increase in numbers of apoptotic cells immunohistochemically in contrast and that these events can be decreased with ferulic acid treatment. In the trauma model created experimentally, we observed that ferulic acid administration causes a decrease in the levels of free radicals as a response to this damage, and a positive effect in oxidant/antioxidant balance developed due to the damage, by increasing antioxidants such as SOD, and GSH. We have detected that ferulic acid which is a strong antioxidant reduced the pathological processes occurring after trauma that was created in the brain.

\section{Conclusion}

This information, and findings indicate that ferulic acid administration in experimentally created brain damage in rats can be a promising treatment in the future.

\section{References}

1. Toklu HZ, Hakan T, Biber N, Solakoglu S, Ögünc AV, Şener G. The protective effect of alpha lipoic acid against traumatic brain injury in rats. Free Radic Res 2009; 43 (7): 658-667.

2. Vural M, Berker E. Clinical Diagnosis and Evaluation in Traumatic Brain Injury. Bakirköy Med J 2012; 8: 53-59.

3. Nichols J, Perez R, Wu C, Adelson PD, Anderson T. Traumatic Brain Injury Induces Rapid Enhancement of Cortical Excitability in Juvenile Rats. CNS Neurosci Ther 2015; 21 (2): 193-203. doi.wiley.com/10.1111/cns. 12351

4. Sandel ME, Bell KR, Michaud LJ. Brain injury rehabilitation. 1. Traumatic brain injury: Prevention, pathophysiology, and outcome prediction. Arch Phys Med Rehabil. 1998; 79 (3 SUPPL. 1): S3-9.

5. Whyte J, Hart T, Laborde A, Rosenthal M. Rehabilitation of the patient with traumatic brain injury. In: Whyte J, Hart T, Laborde A, Rosenthal M, editors. Rehabilitation medicine : principles and practice. Philadelphia, PA: Lippincott Williams \& Wilkins, a Wolters Kluwer; 1998: 1191-1239.

6. Mayeux JP, Teng SX, Katz PS, Gilpin NW, Molina PE. Traumatic brain injury induces neuroinflammation and neuronal degeneration that is associated with escalated alcohol self-administration in rats. Behav Brain Res 2015; 279: 22-30.

7. Sharp DJ, Beckmann CF, Greenwood R, Kinnunen KM, Bonnelle $\mathrm{V}$, De Boissezon $\mathrm{X}$ et al. Default mode network functional and structural connectivity after traumatic brain injury. Brain 2011; 134 (8): 2233-2247.

8. Warner MA, de la Plata CM, Spence J, Wang JY, Harper C, Moore C et al. Assessing Spatial Relationships between Axonal Integrity, Regional Brain Volumes, and Neuropsychological Outcomes after Traumatic Axonal Injury. J Neurotrauma 2010; 27 (12): 2121-2130. www.liebertonline.com/ doi/abs/10.1089/neu.2010.1429

9. Xu Y, McArthur DL, Alger JR, Etchepare M, Hovda DA, Glenn TC, et al. Early nonischemic oxidative metabolic dysfunction leads to chronic brain atrophy in traumatic brain injury. J Cereb Blood Flow Metab 2010; 30 (4): 883-894.

10. Robertson CL, Scafidi S, McKenna MC, Fiskum G. Mitochondrial mechanisms of cell death and neuroprotection in pediatric ischemic and traumatic brain injury. Exp Neurol 2009; 2018: 371-380.

11. Ozbal S, Cankurt U, Tugyan K, Pekcetin C, Sisman AR, Gunduz $\mathbf{K}$ et al. The effects of $\alpha$-lipoic acid on immature rats with traumatic brain injury. Biotech Histochem 2015; 90 (3): 206-215. www.ncbi.nlm.nih.gov/ pubmed/25420894

12. Ojha S, Javed H, Azimullah S, Khair SBA, Haque ME. Neuroprotective potential of ferulic acid in the rotenone model of Parkinson's disease. Drug Des Devel Ther 2015; 9: 5499-5510.

13. Sung JH, Gim SA, Koh PO. Ferulic acid attenuates the cerebral ischemic injury-induced decrease in peroxiredoxin- 2 and thioredoxin expression. Neurosci Lett 2014; 566:88-92.

14. Koh PO. Ferulic acid attenuates focal cerebral ischemia-induced decreases in p70S6 kinase and S6 phosphorylation. Neurosci Lett 2013; 555: 7-11. 
15. Zhao Z, Egashira Y, Sanada H. Ferulic acid is quickly absorbed from rat stomach as the free form and then conjugated mainly in liver. J Nutr 2004; 134 (11): 3083-3088.

16. Mancuso C, Santangelo R. Ferulic acid: Pharmacological and toxicological aspects. Food Chem Toxicol 2014; 65: 185-195.

17. Uchiyama T, Miyazaki K, Yaoi K. Characterization of a Novel $\beta$-Glucosidase from a Compost Microbial Metagenome with Strong Transglycosylation Activity. J Biol Chem [Internet] 2013; 288 (25): 18325-18334. www.jbc.org/lookup/doi/10.1074/jbc.M113.471342

18. Paglia DE, Valentine WN. Studies on the quantitative and qualitative characterization of erythrocyte glutathione peroxidase. J Lab Clin Med 1967; 70 (1): 158-169.

19. Lowry OH, Rosebrough NJ, Farr AL, Randall RJ. Protein measurement with the Folin-Phenol Reagent. J Biol Cem 1951; 193 (1): 265-275. http://linkinghub.elsevier.com/retrieve/pii/S0003269784711122

20. Davis AE. Mechanisms of traumatic brain injury: Biomechanical, structural and cellular considerations. Crit Care Nurs Q 2000; 23 (3): 1-13.

21. Draper HH, Hadley M. Malondialdehyde determination as index of lipid Peroxidation. Methods Enzymol. 1990; 186 (C): 421-431.

22. Girotti AW. Lipid hydroperoxide generation, turnover, and effector action in biological systems. J Lipid Res 1998; 39:1529-42.
23. Saba M, Morales CR, De Lamirande E, Gagnon C. Morphological and biochemical changes following acute unilateral testicular torsion in prepubertal rats. J Urol 1997; 157 (3): 1149-1154. www.ncbi.nlm.nih. gov/pubmed/9072560

24. McCord JM, Edeas MA. SOD, oxidative stress and human pathologies: a brief history and a future vision. Biomed Pharmacother 2005; 59 (4): 139-142. http://linkinghub.elsevier.com/retrieve/pii/S0753332205000387

25. Tugyan K, Ozbal S, Cilaker S, Kiray M, Pekcetin C, Ergur BU et al. Neuroprotective effect of erythropoietin on nandrolone decanoate-induced brain injury in rats. Neurosci Lett 2013; 533: 28-33. http://linkinghub.elsevier.com/retrieve/pii/S0304394012013171

26. Sönmez U, Sönmez A, Erbil G, Tekmen I, Baykara B. Neuroprotective effects of resveratrol against traumatic brain injury in immature rats. Neurosci Lett 2007; 420 (2): 133-137. www.ncbi.nlm.nih.gov/pubmed/17531385

27. Krbila S, Waczulikova I, Sobona V, Zahorec R. Impact of intracranial pressure measurement on survival in patients with severe traumatic brain injury. Bratisl Lek Listy 2013; 114 (12): 696-701.

28. Sivak S, Nosal V, Bittsansky M, Dluha J, Dobrota D, Kurca E. Type and occurrence of serious complications in patients after mild traumatic brain injury. Bratisl Lek Listy 2016; 117 (1): 22-25.

Received February 18, 2019. Accepted March 18, 2019. 